\title{
High Energy Laser Systems for Short Range Defense
}

\author{
M. LAVAN* \\ United States Army Space and Missile Defense Command, U.S. Army Forces Strategic Command \\ The advantages of the use of high energy laser systems in a counter rocket, artillery and mortar projectile \\ mission are described. The history of the development of directed energy weapons in the United States Army and \\ the current high energy laser programs and technologies are outlined. The U.S. Army vision for future ground \\ combat relies on high mobility, lightweight systems with improved survivability. Without the protection afforded \\ by heavy armor, other methods for protecting assets and personnel are required. The Army is now developing the \\ technologies for a future mobile solid state laser weapon system to defeat a wide range of air and ground threats, \\ working to get these revolutionary weapons to our soldiers in the near future.
}

PACS numbers: 75.47.Lx, 75.47.Gk, 73.50.Fq

\section{Introduction}

The destructive power and accuracy of today's conventional and unconventional weapon systems threaten the survivability of NATO forces. Defeating these increasingly lethal threats will require new and revolutionary capabilities for our armed forces. The U.S. Army has embarked on a program to develop and test high energy laser (HEL) technologies and provide these new weapon capabilities to our commanders on the battlefield.

The Army believes that high energy laser weapons can be effectively employed in a variety of mission areas, providing unique or complementary capabilities to other weapon systems and resolving several existing capability gaps identified by the soldier in the field:

- Counter rocket, artillery, and mortar (CRAM);

- Defeat man-portable surface-to-air missiles in flight;

- Standoff mine neutralization and explosive ordnance disposal;

- Standoff counter improvised explosive device;

- Counter anti-tank guided missile;

- Anti-rocket propelled grenade;

- Disrupt or defeat electro-optic or infrared sensors;

- Ultra-precision strike - kill or disable targets with no (or minimal) collateral damage.

\footnotetext{
* corresponding author; e-mail: michael.j.lavan@us.army.mil
}

HEL weapons offer the unique performance capabilities of near real-time engagements of targets, scalable lethality effects, and precision engagements with minimal collateral effects:

- Precision application of energy;

- Graduated response;

- Depth of magazine.

- Operation at the speed of light;

- Low cost per kill.

The U.S. Army Space and Missile Defense Command/US Army Forces Strategic Command (SMDC/ARSTRAT) is the lead technical agency within the Army responsible for developing high power directed energy (DE) technologies and capabilities. Along with other Department of Defense (DoD) organizations, SMDC/ARSTRAT has over the last thirty years demonstrated the capability of DE technologies to defeat a wide variety of threats. The two largest programs, the Ground Based Free Electron Laser Technology Integration Experiment and the Neutral Particle Beam Program, were executed primarily in the 1980 s and were intended to develop the technologies required for destroying strategic ballistic missiles. As the emphasis shifted in the early 1990s from defeating strategic threats to theater ballistic missiles threats, SMDC/ARSTRAT's DE technology development efforts also changed to match this more immediate threat.

In 1990, SMDC assumed the responsibility for operating and maintaining the High Energy Laser System Test Facility (HELSTF) at White Sands Missile Range in New Mexico. In 1996, SMDC conducted a major DE technology demonstration at HELSTF using the high power deuterium fluoride (DF) mid-infrared advanced chemical laser (MIRACL) by successfully destroying a 
short-range rocket in flight. This demonstration provided strong evidence of the potential of $\mathrm{DE}$ systems to meet evolving air and missile defense requirements. This occurred during the "Nautilus" lethality demonstration program, a collaborative effort with the Government of Israel. The Nautilus program demonstrated a capability previously unavailable to the warfighter: active defense against the threat from ever-increasingly accurate and numerous rockets and artillery projectiles.

\section{Laser Technology Development}

The Army successfully developed the Tactical High Energy Laser (THEL) Advanced Concept Technology Demonstration for the CRAM mission, but it ceased activity in DF laser development in September 2005, for a variety of reasons:

- To fund other, higher priority requirements;

- Israel reduced its funding commitment to a mobile THEL;

- Large physical size of DF laser compared to a (1.06 $\mu \mathrm{m}$ wavelength) solid state laser (SSL);

- Avoid release of noxious effluents from DF to the atmosphere;

- Avoid storage and handling of hazardous DF fluids in highly pressurized tanks;

- Reduce cost per kill for laser consumables;

- Reduce logistics supportability problems with unique DF chemicals.

Rapid development of the SSL offered a better solution for battlefield applications of high energy lasers. The technology program was driven by the multi-service DoD organization High Energy Laser Joint Technology Office (HEL JTO). Its Joint High Power Solid State Laser (JHPSSL) program is a key enabler for future SSL weapon development by the Army, Navy, and Air Force.

The basic elements of an HEL weapon system are shown in Fig. 1. The development risks for many of these elements have been or will be mitigated by previous, ongoing, or planned HEL development programs.

It is envisioned that an HEL weapon system will receive target tracks from a network of external sensors such as the AN/MPQ-64 Sentinel radar shown. The key challenges for handing-off targets to the HEL weapon are achieving the position accuracy and update rate required to assure acquisition by the laser system's coarse acquisition optical sensor at an adequately long range. SMDC demonstrated this can be successfully accomplished during the THEL program.

The laser diodes will be pumped by electrical energy that will be stored in a battery pack or other type of storage device. We must now develop DC converters to

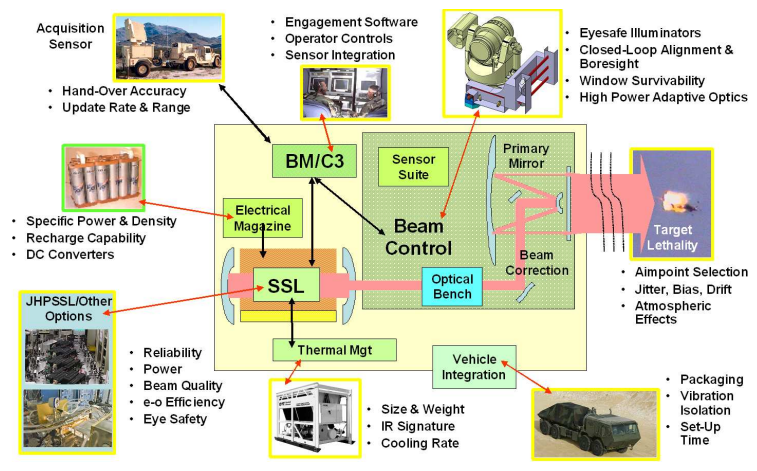

Fig. 1. HEL weapon technology.

control the voltage and current to diodes, are reliable in a military environment, and small in size and weight. We must also be able to recharge the system to meet expected HEL weapon system operational availability requirements.

The SSL that will be used in the HEL weapon system may be the type that is being developed on JHPSSL or may be from other sources. Beam power, electro-optical efficiency and run time are key drivers for determining the size and weight of an SSL system. The smaller the power and the shorter the required run time, the smaller the thermal management system needs to be. We will evaluate alternative cooling technologies and work with other Defense agencies and industry to develop systems which meet our needs. The size of the cooling system will also be a major factor in the selection of the vehicle platform. Shown is a hybrid electric version of the heavy expanded mobility tactical truck (HEMTT). However, we are considering other platforms as our laser device technologies mature. Ideally the objective HEL weapon system will be deployable in a smaller tactical vehicle.

During THEL, the Army successfully demonstrated the software and control hardware necessary to engage RAM targets. This software will be refined during the High Energy Laser Technology Demonstrator (HEL TD) program. We also successfully demonstrated many of the components for a Beam Control System during THEL. However, we are continuing efforts to decrease the size and weight of the system and improve such elements as the illuminators, the exit window, and to provide for a future adaptive optics system to correct atmospheric effects. We are developing an optics alignment and boresighting capability to improve significantly the operational set-up time. We will also have to limit the jitter effects if we are to take advantage of the shorter wavelength and smaller diameter beam director compared to a DF system. Many of these elements are boxed in yellow, designating them as moderate risks for one $\mu \mathrm{m}$ HEL programs.

Through the JHPSSL program, SMDC/ARSTRAT is leading the development of two alternative SSL laboratory devices, in conjunction with the HEL JTO, Army Research Laboratory, Air Force Research Laboratory, 
and Office of Naval Research. The HEL JTO initiated the JHPSSL program and has continued to provide much of the funding and many of the ideas as JHPSSL has progressed. The goals of the initial phases of the JHPSSL program were to develop $25 \mathrm{~kW}$-class laboratory devices with near diffraction-limited beam quality and long run time. The results of those efforts gave us the confidence to continue to develop weapon-class SSLs. The goal of the current phase of the JHPSSL program is to develop and demonstrate alternative $100 \mathrm{~kW}$-class, diodepumped solid-state lasers.

The Army Science and Technology (S\&T) program includes the HEL TD program to integrate an SSL, beam control, electrical power supply, thermal management, fire control and communications elements on a wheeled tactical platform and demonstrate in a relevant operational environment its capability to counter RAM targets. Countering the RAM threat requires high power and excellent beam quality to provide effective area coverage against this threat-class due to their speed and trajectory characteristics. The Army S\&T Program is also conducting basic and applied research on enabling technologies that will support future enhancements to the performance of SSL weapon systems.

\section{JHPSSL}

A major purpose of the JHPSSL program is to develop SSL technologies with performance and packaging capabilities suitable for future integration as elements of the Future Combat System. The Phase 2 JHPSSL program goal was to demonstrate a laser with an average power of greater than $25 \mathrm{~kW}$, high beam quality, long run time, short start-up time, high wall-plug efficiency, high output power-to-mass ratio, and low beam jitter. This phase concluded with independent government testing at multiple locations in 2005.

The Phase 3 program objective is to develop $100 \mathrm{~kW}$ class, diode-pumped SSL laboratory device(s) with excellent beam quality relative to the diffraction limit (DL). Emphasis is being placed on laser architectures that the government believes are favorable in terms of size, weight, efficiency, reliability, maintainability, supportability, environmental acceptability, and ruggedness. The most important performance Phase 3 parameters are beam quality, average output power, run time, and electro-optical efficiency. These will be demonstrated by direct physical measurements except efficiency, which can be derived based on physical measurements and analysis.

- The instantaneous laser beam quality threshold shall be $2 \times \mathrm{DL}$ with a goal of $1.5 \times \mathrm{DL}$, based on a power-in-the-bucket measurement in the far field.

- The bulk SSL average output beam power shall be at least $100 \mathrm{~kW}$ at a wavelength that is highly transmissive through the atmosphere.

- The laboratory laser device run-time threshold shall support the ability to produce three 5-second shots during the first $20 \mathrm{~s}$ of a 200-second engagement sequence, followed by seven additional 5 second shots at a semi-sustained duty cycle of $20 \%$. The duty cycle includes consideration of the time to negate the target with the laser heating, the retarget time, and the waste heat elimination time. The laser device run-time goal is five 5 -second shots during the first $33 \mathrm{~s}$ of a 300 -second engagement sequence, followed by thirteen additional 5 -second shots at a semi-sustained duty cycle of $25 \%$.

- The threshold electro-optical efficiency is $17 \%$ with a goal of $19 \%$. This efficiency is the output laser optical power divided by the input electrical power provided to the laser diodes. The threshold and goal efficiencies are based on an expected diode array efficiency of $50 \%$. This assumes that all photons from the diode lasers are counted, both spatially and spectrally. Contractor(s) are required to measure their average diode array efficiency. If this measured diode array efficiency is greater or less than the $50 \%$, the threshold and goal for the overall efficiency will be linearly adjusted.

Two Phase 3 development contract awards were awarded in December 2005, to Northrop Grumman Space Technology (NGST) and Textron. Their approaches are outlined in Fig. 2. The NGST approach involves:

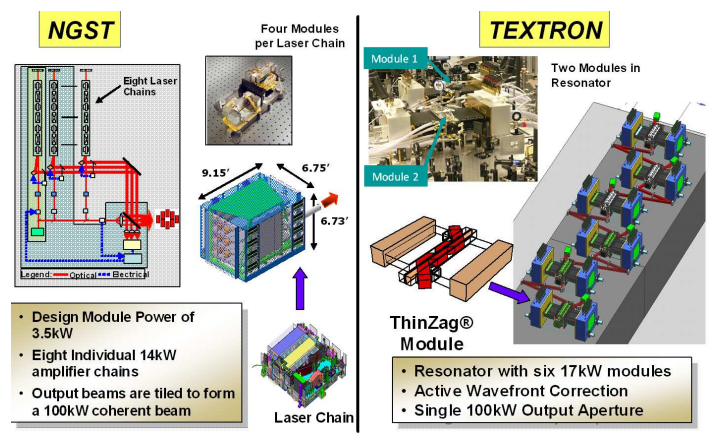

Fig. 2. JHPSSL architectures.

- Single low-power master oscillator injecting multiple amplifier chains;

- Master oscillator power amplifier (MOPA) outputs wavefront corrected, coherently combined, and stacked side-by-side to form a common beam. The $15 \mathrm{~kW}$ laser chain performance was initially demonstrated on a separate program called Vesta;

- Eight laser chains integrated into a single unit with a beam combiner subsystem.

The Textron approach is based on their ThinZag ${ }^{\mathrm{TM}}$ design using Nd:YAG ceramic slabs that can potentially scale to very high power in a single aperture: 
- No multiple beams; no "beam combining" (alignment ease);

- No optical phasing of multiple chains;

- No MOPA with resulting numerous apertures;

- ThinZag@ Ceramic Laser Modules present a path to $100 \mathrm{~kW}$ that has a low part count.

\section{HEL TD}

The HEL TD program objective is to demonstrate in a relevant operational environment at HELSTF that a mobile SSL weapon system can provide an effective CRAM mission capability. Contracts were awarded to Boeing and Northrop Grumman in 2007.

The HEL TD will support the transition to a formal Army weapon system acquisition program. It will provide adequate output laser power to demonstrate lethality against RAM targets and address other capability gaps. It will have variable output power so that the effects can be more controlled. It will be road-mobile, potentially deployable by air, land and sea. The laser, BCS, power supply, thermal management systems and BMC3 elements will be integrated. The system will have interfaces with external tactical surveillance and acquisition sensors and tactical communication links with higher echelon command.

The acquisition strategy is phased:

- Phase I, basic contract. Design a ruggedized BCS, culminating at preliminary design review.

- Phase II, BCS completion option. Complete the design and fabrication of a ruggedized HEL TD BCS, integrate it on a tactical wheeled vehicle, and test (pointing and tracking) at HELSTF.

As an option to the JHPSSL Phase 3 contracts, Textron and NGST were funded to conduct systems engineering efforts to model the military utility of an HEL weapon system based on their respective JHPSSL technologies. The government provided threat scenarios, and the contractors evaluated the performance of a ruggedized version of their SSL or one with similar characteristics. Also, included in the analysis were an evaluation of technology shortcomings, a rigorous development cost estimate, and the definition of multiple configurations based on alternative thermal management, electrical power, and beam control subsystems.

\section{Technology Issues}

Atmospheric turbulence causes laser beam spreading, wander, intensity fluctuations, and beam break-up. Mitigation has not yet been demonstrated in a one $\mu \mathrm{m}$ HEL system. "Target Loop Adaptive Optics" (AO) refers to a closed loop system designed to detect and correct the phase variations in a wavefront. The fundamental idea is to form a conjugate of the aberrated wavefront on the deformable mirror (DM), flattening the wavefront downstream.

One of the most common AO architectures includes:

- Actuator-based DM. Xinetics builds commercial DMs with 37 to 941 actuators.

- Hartmann wavefront sensor (WFS) lenslets divide the wavefront into "sub-apertures." Each subaperture area on the focal plane is composed of many pixels. By finding the intensity on each, the centroid moment of the illumination pattern may be computed.

- Matrix reconstructor takes the measured subaperture tilts and finds the corresponding actuator commands.

- Tip-tilt mirror is often used to off-load overall tilt from the DM.

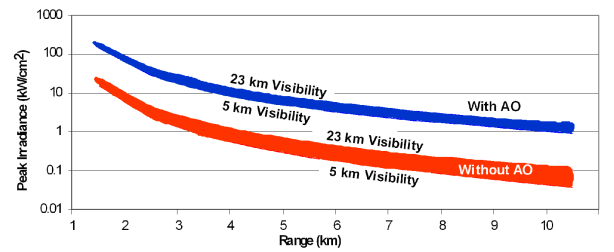

Fig. 3. HEL beam propagation. HELCOMES Engagement Code, Dr. Richard St. John, SAIC. Adaptive optics: $0.35 \mathrm{~cm}$ actuator spacing on $50 \mathrm{~cm}$ diameter primary, 160 actuators over $5 \mathrm{~cm}$ diameter optics. Common factors: $45^{\circ}$ elevation angie, $C_{n}^{2}=10^{13} \mathrm{~m}^{-2 / 3}$, cross wind $=9 \mathrm{~m} / \mathrm{s}$.

From Fig. 3, to achieve a peak irradiance of $1 \mathrm{~kW} / \mathrm{cm}^{2}$, with the low visibility atmospheric model (bottom of the bands), without AO (the bottom band) the target must be at a range of $2.8 \mathrm{~km}$. With $\mathrm{AO}$ (top band), the range could be $9 \mathrm{~km}$. HELCOMES, used to generate theses curves, is an HEL system-level engagement code anchored to SAIC's wave optics code, atmospheric compensation simulation (ACS).

One serious concern with a kinetic energy intercept of a RAM target is that any high-acceleration movement by the target, whether intentional or not, requires that the interceptor have an effective acceleration capability that is at least several times higher. A target might maneuver at up to $50 \mathrm{G}$, obviously presenting a serious challenge to the interceptor. But such a maneuvering target should be readily handled by an HEL system. For instance, even at the short range of $1 \mathrm{~km}$, a $50-\mathrm{G}$ maneuver will change the angular motion in $2 \mathrm{~ms}$ by about $1 \mu \mathrm{rad}$. The laser tracking system will operate at a track loop bandwidth of $500 \mathrm{~Hz}$ or greater, and position measurements of the target are taking place at $5000 \mathrm{~Hz}$ or greater. The 
$1 \mu \mathrm{rad}$ displacement is, worst case, as large as a single pixel in the fine tracking camera. It is unlikely for the camera to use much better than a $1 \mu$ rad resolution for a tactical system due to diffraction. Thus it is fairly easy for the directed energy tracking system to keep up with this maneuvering target, and also fairly easy for the HEL mirror system gimbals and fast steering mirror to make the engagement. The main gimbals accelerate at about $5 \mathrm{rad} / \mathrm{s}^{2}=10 \mu \mathrm{rad}$ in $2 \mathrm{~ms}$. Fast steering mirror bandwidths of $2 \mathrm{kHz}$ are available as commercial off-the-shelf items.

Lethality is the science of understanding the process by which a DE weapon can defeat a target, and the translation of that understanding into tools, methods, databases and supporting infrastructure needed to perform quantitative assessments of DE weapon effectiveness. The lethality program develops the lethality criteria: the beam-on-target conditions (energy fluence, spotsize, duration, etc.) necessary to achieve a specific degradation in the ability of a threat target system or munition to complete its designated mission. Lethality criteria are developed for feasible kill mechanisms against selected threats, giving preference to the RAM threat set and to target failure modes with observable kill signatures. These lethality criteria, incorporated into weapon effectiveness tables and algorithms, provide lethality input to the higher-operational-level modelling and simulation required for system optimization and military utility analysis.

In the Rapid Cook-Off (RCO) kill mechanism, laser radiation heats the threat projectile casing, and the heat then conducts through the metal to the explosive charge. That surface ignites, creating great pressure which ruptures the casing. This mechanism has been well-characterized in the laboratory, in full-scale static tests, and in dynamic flight tests using THEL.

\section{Summary}

The U.S. Army vision for future ground combat relies on high mobility, lightweight systems with improved survivability. Without the protection afforded by heavy armor, other methods for protecting assets and personnel are required. The Army successfully demonstrated a DF HEL system against RAM targets and will demonstrate a $100 \mathrm{~kW}$ SSL in the laboratory in 2009. It is now developing the HEL technologies for a future mobile SSL weapon system to defeat a wide range of air and ground threats, working to get these revolutionary weapons to our soldiers in the near future. 